\title{
Emergence of four cases of KPC-2 and KPC-3-carrying Klebsiella pneumoniae introduced to Switzerland, 2009-10
}

B Babouee ${ }^{1}$, A F Widmer ${ }^{1}$, O Dubuis², D Ciardo², S Droz ${ }^{3}$, B Y Betsch ${ }^{4}$, C Garzoni $^{4}$, U Führer ${ }^{4}$, M Battegay ${ }^{1}$, R Frei ${ }^{5}$, D Goldenberger (dgoldenberger@uhbs.ch) 5

1. Division of Infectious Diseases and Hospital Epidemiology, University Hospital Basel, Basel, Switzerland

2. Viollier AG, Basel, Switzerland

3. Institute for Infectious Diseases, University of Berne, Berne, Switzerland

4. University Clinic for Infectious Diseases, University Hospital and University of Berne, Inselspital, Berne, Switzerland

5. Division of Clinical Microbiology, University Hospital Basel, Basel, Switzerland

Citation style for this article:

Babouee B, Widmer AF, Dubuis O, Ciardo D, Droz S, Betsch BY, Garzoni C, Führer U, Battegay M, Frei R, Goldenberger D. Emergence of four cases of KPC-2 and KPC3-carrying Klebsiella pneumoniae introduced to Switzerland, 2009-10. Euro Surveill. 2011;16(11):pii=19817. Available online: http://www.eurosurveillance.org/ ViewArticle.aspx?Articleld $=19817$

Article published on 17 March 2011

We report four epidemiologically unrelated cases of KPC-carrying Klebsiella pneumoniae identified in Switzerland between May 2009 and November 2010. Three cases were transferred from Italy (two KPC-3, one KPC-2) and one from Greece (KPC-2). Resistance to colistin and doxycycline emerged in one KPC-3carrying $K$. pneumoniae strain during therapy. These results demonstrate ongoing dissemination of KPC throughout Europe. Rapid and reliable identification of KPC and implementation of control measures is essential to limit spread.

\section{Introduction}

Carbapenems are first-line drugs for severe infections caused by Enterobacteriaceae expressing extendedspectrum beta-lactamases (ESBLs). The emergence of carbapenemase-producing Enterobacteriaceae in the past years is of great concern [1]. After the characterisation of the first Klebsiella pneumoniae isolate producing carbapenemase of the KPC type in 1996 in the United States [2], the KPC-producing bacteria spread worldwide. Poirel et al. reported the first KPC-2 $K$. pneumoniae isolate in Switzerland imported from Sicily (Italy) in 2010 [3]. Here, we report four additional imported cases of KPC-carrying K. pneumoniae detected in Switzerland.

\section{Materials and methods}

The four KPC-suspected strains were collected from different Swiss hospitals and were sent to our laboratory in Basel for confirmation. We performed conventional susceptibility testing using an automated micro-dilution test system (Micronaut-S, MERLIN Diagnostika $\mathrm{mbH}$ ).Determination of minimum inhibitory concentrations (MIC) was performed with Etest stripes (bioMérieux). Results were interpreted according to the European Committee on Antimicrobial Susceptibility Testing (EUCAST) guidelines [4], except for doxycycline which was interpreted according to the guidelines of Clinical and Laboratory Standards Institute (CLSI) [5].

For phenotypic identification of KPC-producing isolates, both double disc synergy test and modified Hodge test were applied [1] . KPC-specific PCR and direct sequencing of the region encompassing the main part of the bla $a_{\text {KP }}$ gene (ca. 820 nucleotides, sequenced in both directions) was performed according to Bradford et al. [6]. The KPC type was identified by the BLAST programme from the National Center for Biotechnology Information Web site (http://www.ncbi. nlm.nih.gov/BLAST).

Clinical data were available for two patients and were collected by full chart review.

\section{Results}

Four cases of KPC-carrying $K$. pneumoniae were detected in Switzerland between May 2009 and November 2010, two of type KPC-2 and two of type KPC-3. All patients were between 50 and 70 years-old and had been transferred from abroad, three from Italy and one from Greece. Two patients were colonised and two were infected with KPC-producing $K$. pneumoniae (Table 1).

The antimicrobial susceptibility profiles of the four K. pneumoniae isolates are listed in Table 2. Double disc synergy test as well as the modified Hodge test showed positive results for KPC-production in all four cases.

Case 1

The patient in their 50 s lived in Greece and travelled to Switzerland in September 2009. The person was colonised in the urine, inguinal and perineum with 
K. pneumoniae bla $a_{\mathrm{KPC}-2}$. No further clinical data were available for this patient.

\section{Case 2}

The patient was in their 6os and hospitalised in Italy due to upper gastro-intestinal problems, and underwent diagnostic endoscopy. The clinical history did not mention any antibiotic treatment. In May 2010 the patient was transferred to Switzerland. On hospital admission a constricted bile duct was stented endoscopically. Because the patient developed fever during the initial empiric antibiotic therapy with piperacillin/ tazobactam, the therapy was changed to meropenem. During treatment with meropenem the patient developed bloodstream infection with $K$. pneumoniae. The isolate was resistant or intermediately resistant to all

TABLE 1

Main characteristics of four cases of KPC-producing K. pneumoniae detected in Switzerland, May 2009 and November 2010

\begin{tabular}{|l|c|c|c|c|}
\hline & Case 1 & Case 2 & Case 3 & Case 4 \\
\hline Age group (years) & $50-59$ & $60-69$ & $40-49$ & No-69 \\
\hline Date of KPC detection & September 2009 & July 2010 & September 2010 & November 2010 \\
\hline Infected/colonised with K. pneumoniae & Colonised & Infected & Infected & Colonised \\
\hline Transferred from & Greece & Italy $^{\mathrm{a}}$ & Italy $^{\mathrm{a}}$ & Italy $^{\mathrm{a}}$ \\
\hline Hospitalised in Switzerland & Eastern part & North-western part & Western part & North-eastern part \\
\hline bla $_{\mathrm{KPC}}$ gene & bla $_{\mathrm{KPC}-2}$ & bla $_{\mathrm{KPC}-3}$ & bla $_{\mathrm{KPC}-2}$ & bla $_{\mathrm{KPC}-3}$ \\
\hline
\end{tabular}

KPC: Klebsiella pneumoniae carbapenemase.

a The three patients were transferred from three different regions in Italy: Apulia, Liguria and Sicily

\section{TABLE 2}

Antimicrobial susceptibility profiles of the four Klebsiella pneumoniae first isolates carrying $b l a_{\mathrm{KPC}-2}$ or bla $a_{\mathrm{KPC}-3}$, Switzerland, May 2009 and November 2010

\begin{tabular}{|c|c|c|c|c|}
\hline Antibiotic & $\begin{array}{l}\text { Case } 1 \\
\text { bla }_{\mathrm{KPC} \cdot 2}\end{array}$ & $\begin{array}{l}\text { Case } 2 \\
\text { bla }_{\mathrm{KPC} .3}\end{array}$ & $\begin{array}{l}\text { Case } 3 \\
b l a_{\mathrm{KPC} \cdot 2}\end{array}$ & $\begin{array}{l}\text { Case } 4 \\
\text { bla }_{\mathrm{KPC} \cdot 3}\end{array}$ \\
\hline Ampicillin & $R$ & $\mathrm{R}$ & $\mathrm{R}$ & $R$ \\
\hline Amoxicillin/clavulanate & $R$ & $R$ & $R$ & $R$ \\
\hline Piperacillin/tazobactam & $R$ & $R$ & $R$ & $R$ \\
\hline Cefuroxime & $R$ & $R$ & $\mathrm{R}$ & $R$ \\
\hline Ceftazidime & $R$ & $R$ & $\mathrm{R}$ & $R$ \\
\hline Ceftriaxone & $R$ & $R$ & $R$ & $R$ \\
\hline Cefepime & $\mathrm{R}$ & $R$ & $R$ & $R$ \\
\hline Aztreonam & $R$ & $R$ & $R$ & $R$ \\
\hline Imipenem & $\mathrm{R}(132)$ & $R(132)$ & $R(16)$ & $R(132)$ \\
\hline Meropenem & $\mathrm{R}(132)$ & $R(132)$ & $R(12)$ & $R(132)$ \\
\hline Ertapenem & $\mathrm{R}(132)$ & $\mathrm{R}(132)$ & $\mathrm{R}(16)$ & $\mathrm{R}(132)$ \\
\hline Trimethoprim/sulfamethoxazole & $R$ & $R$ & $\mathrm{R}$ & $R$ \\
\hline Nitrofurantoin & $R$ & $R$ & ND & $R$ \\
\hline Norfloxacin & $\mathrm{R}$ & $\mathrm{R}$ & ND & $R$ \\
\hline Ciprofloxacin & $R(132)$ & $R(132)$ & $R(132)$ & $R(132)$ \\
\hline Levofloxacin & $R$ & $R$ & ND & $R$ \\
\hline Gentamicin & S (1) & $S(2)$ & $S(2)$ & $I(4)$ \\
\hline Tobramycin & ND & 1 & $R$ & ND \\
\hline Amikacin & $\mathrm{S}$ & $\mathrm{S}$ & $R$ & ND \\
\hline Netilmicin & $\mathrm{R}$ & $R$ & $R$ & ND \\
\hline Colistin & $R(4)$ & $\begin{array}{c}\mathrm{S}(0.25)^{\mathrm{a}} \\
\mathrm{R}(16)^{\mathrm{b}}\end{array}$ & $S(0.25)$ & $S(0.5)$ \\
\hline Doxycycline & $S(4)$ & $\begin{array}{c}S(4)^{a} \\
R(16)^{b}\end{array}$ & I (8) & I (8) \\
\hline Tigecycline & S (1) & I (2) & $\mathrm{S}(1)$ & I (2) \\
\hline Fosfomycin & $\mathrm{S}(32)$ & $R$ (128) & $S(32)$ & $R$ (128) \\
\hline
\end{tabular}

I: intermediate; ND: no data; R: resistant; S: susceptible.

Minimum inhibitory concentration in $\mathrm{mg} / \mathrm{L}$ is given in parentheses.

a Before antimicrobial treatment.

${ }^{\mathrm{b}}$ After antimicrobial treatment. 
antibiotics tested (see Table 2) except gentamicin, colistin, and doxycycline. KPC-specific PCR was performed detecting bla $_{\mathrm{KPC}-3}$. The antibiotic therapy was adapted to intravenous colistin and gentamicin. Repeated stenosis of the intra-ductal stent and suspicion of cholangiocarcinoma provided evidence for a Whipple operation with pancreaticogastrostomy. After the operation the patient developed acute arterial bleeding and had to be surgically revised. Shortly after the second operation he had relapsing bloodstream infection with K. pneumoniae which were newly resistant to colistin. Colistin treatment was stopped and doxycycline and later fosfomycin were added to gentamicin. Fosfomycin was given because routine testing revealed an intermediate result. The patient died from multiorgan failure caused by an uncontrolled infection complicated by intra-abdominal bleeding. A bronchoalveolar lavage specimen taken one day before death was positive for K. pneumoniae, now also resistant to doxycycline.

\section{Case 3}

The patient in their 40 s was injured in July 2009 in Italy. The person was treated initially with an external fixation for a pelvic fracture in a primary care hospital in Italy and referred to the University Hospital of Berne for secondary care. The surgeon noted a pin track infection and performed a wound excision. Perioperatively, as well as at discharge, no signs of infection were present. In September 2010 the patient was readmitted because of a pelvic abscess, which was drained and osteosynthesis material was removed. Empiric therapy included amoxicillin/clavulanate, but cultures of the abscess revealed a K. pneumoniae isolate resistant to all antibiotics tested except gentamicin, colistin, tigecycline and fosfomycin (Table 2).

Antibiotic treatment was switched to tigecycline and gentamicin, followed by a second and third surgical debridement. After two weeks gentamicin was discontinued because of ototoxicity and therapy with tigecycline for a chronic osteomyelitis was continued for three months. To the best of our knowledge the patient was cured with this antibiotic course.

\section{Case 4}

The patient was in their 6os and hospitalised in an intensive care unit in Italy for one month because of a cerebral vascular insult, before being transferred to Switzerland. This patient was colonised in the upper respiratory tract and on the subclavian catheter with $K$. pneumoniae that contained the bla $a_{\mathrm{KPC}-3}$ gene.

\section{Infection control measures}

Cases 2 and 3 were isolated immediately after detection of KPC-producing $K$. pneumoniae by contact isolation $[5,6]$, which involved stay in a single-patient room, use of gloves and gowns by medical personnel during physical contact, and use of masks by medical personnel when exposure to respiratory secretions was expected. Secondary spread was investigated by active surveillance. This included screening of all patients sharing the same room as the index patient for ESBL or KPC by testing stool samples and, if applicable, samples from wounds, drainages, etc. None of these patients was found to be positive for KPCcarrying Enterobacteriaceae. Patients who stayed in the same room as the index patient and who were already discharged from hospital were tagged in the hospital administration system to be screened at the next admission. Infection control measures taken around Cases 1 and 4 could not be made available during this study.

\section{Discussion and conclusions}

The global dissemination of KPC-producing K. pneumoniae is of great concern to public health services worldwide [9]. The first outbreak outside the United States was documented in Israel in 2004 [10]. Several reports of outbreaks and sporadic cases of KPC-producing

K. pneumoniae in Italy and Greece have been described and indicated rapid spread within these two countries [11-15]. On the epidemiological scale for spread of KPC-carrying K. pneumoniae defined by Grundmann et al. [1] Greece was determined in November 2010 as 'endemic' and Italy as a country with 'interregional spread'. The first case of a KPC-2-producing K. pneumoniae in Switzerland was described in 2010 from a patient transferred from Sicily [3].

We document four further cases of KPC-carrying K. pneumoniae introduced to Switzerland, three from Italy and one from Greece. Three patients have been discharged from hospital in a stable state of health, one patient died from uncontrolled infection complicated by arterial intraabdominal bleeding. In one case the KPC-3 carrying $K$. pneumoniae strain developed resistance to colistin and doxycycline despite combined antibiotic treatment.

Phenotypic and molecular tests for KPC detection were concordant and confirmed carbapenemase production in all strains by conventional susceptibility testing. As shown in Table 2, the antibiotic susceptibility patterns of the four independent isolates were similar.

In this study, we confirm continuous dissemination of KPC-producing isolates from Greece and Italy to Switzerland. The fact that the three patients transferred from Italy had been hospitalised in three different geographic regions in Italy, Apulia, Liguria and Sicily, is a further indication for a wider dissemination of these isolates. We found no evidence for local spread within the Swiss hospitals where the four reported cases were treated, but sporadic cases are thought to precede endemicity, a likely scenario for resistance markers encoded on plasmids [9]. The worldwide spread of KPC-carrying K. pneumoniae is worrying, since this species is a main source for hospital-acquired infections in critically ill patients and well known for its ability to transfer resistance determinants. Due to the high mobility of KPC genes we anticipate that KPC-mediated resistance will be a prominent mechanism of multidrug 
resistance in Gram-negative bacilli in the near future. As antimicrobial treatment options are dramatically restricted, rapid and reliable identification of KPC is mandatory to generate concepts to limit spread.

\section{References}

1. Grundmann H, Livermore DM, Giske CG, Canton R, Rossolini GM, Campos J, et al. Carbapenem-non-susceptible

Enterobacteriaceae in Europe: conclusions from a meeting of national experts. Euro Surveill. 2010;15(46): pii=19711. Available from: http://www.eurosurveillance.org/ViewArticle. aspx?Articleld $=19711$

2. Yigit H, Queenan AM, Anderson GJ, Domenech-Sanchez A, Biddle JW, Steward CD, et al. Novel carbapenem-hydrolyzing beta-lactamase, KPC-1, from a carbapenem-resistant strain of Klebsiella pneumoniae. Antimicrob Agents Chemother. 2001;45(4):1151-61.

3. Poirel L, Lienhard R, Potron A, Malinverni R, Siegrist HH, Nordmann P. Plasmid-mediated carbapenem-hydrolysing \{beta\}-lactamase KPC-2 in a Klebsiella pneumoniae isolate from Switzerland. J Antimicrob Chemother. 2011;66(3):675-6.

4. European Committee on Antimicrobial Susceptibility Testing (EUCAST). Breakpoint tables for interpretation of MICs and zone diameters. Version 1.3, 5 Jan 2011. Available from: http:// www.eucast.org/fileadmin/src/media/PDFs/EUCAST_files/ Disk test_documents/EUCAST_breakpoints_v1.3_pdf.pdf

5. Clinical and Laboratory Standards Institute (CLSI). Performance Standards for Antimicrobial Susceptibility Testing, Twenty-First Informational Supplement M100-S21, Vol. 31, No. 1, January 2011. Wayne: CLSI; 2011.

6. Bradford PA, Bratu S, Urban C, Visalli M, Mariano N, Landman D, et al. Emergence of carbapenem-resistant Klebsiella species possessing the class A carbapenem-hydrolyzing KPC-2 and inhibitor-resistant TEM-30 beta-lactamases in New York City. Clin Infect Dis. 2004;39(1):55-60.

7. Marschall J, Mühlemann K. Duration of methicillinresistant Staphylococcus aureus carriage, according to risk factors for acquisition. Infect Control Hosp Epidemiol. 2006;27(11):1206-12.

8. Widmer AF, Ruef C, Troillet N. Neue Isolationsrichtlinien in den USA für Spitäler und andere Gesundheitseinrichtungen: Bedeutung für die Schweiz. [New isolation guidelines in the US for hospitals and other health institutions: implications for Switzerland]. Swiss-NOSO. 2009;15(1). German. Available from: http://www.chuv.ch/swiss-noso/d151a1.htm

9. Nordmann P, Cuzon G, Naas T. The real threat of Klebsiella pneumoniae carbapenemase-producing bacteria. Lancet Infect Dis. 2009;9(4):228-36.

10. Leavitt A, Navon-Venezia S, Chmelnitsky I, Schwaber MJ, Carmeli Y. Emergence of KPC-2 and KPC-3 in carbapenemresistant Klebsiella pneumoniae strains in an Israeli hospital. Antimicrob Agents Chemother. 2007;51(8):3026-9.

11. Cuzon G, Naas T, Demachy MC, Nordmann P. Plasmidmediated carbapenem-hydrolyzing beta-lactamase KPC-2 in Klebsiella pneumoniae isolate from Greece. Antimicrob Agents Chemother. 2008;52(2):796-7.

12. Fontana C, Favaro M, Sarmati L, Natoli S, Altieri A, Bossa MC, et al. Emergence of KPC-producing Klebsiella pneumoniae in Italy. BMC Res Notes. 2010;3:40.

13. Giakoupi P, Maltezou H, Polemis M, Pappa O, Saroglou $\mathrm{G}$, Vatopoulos A, et al. KPC-2-producing Klebsiella pneumoniae infections in Greek hospitals are mainly due to a hyperepidemic clone. Euro Surveill. 2009;14(21):pii=19218. Available from: http://www.eurosurveillance.org/ViewArticle. aspx?Articleld $=19218$

14. Giani T, D’Andrea MM, Pecile P, Borgianni L, Nicoletti P, Tonelli $\mathrm{F}$, et al. Emergence in Italy of Klebsiella pneumoniae sequence type 258 producing KPC-3 carbapenemase. J Clin Microbiol. 2009;47(11):3793-4.

15. Tsakris A, Kristo I, Poulou A, Markou F, Ikonomidis A, Pournaras S. First occurrence of KPC-2-possessing Klebsiella pneumoniae in a Greek hospital and recommendation for detection with boronic acid disc tests. J Antimicrob Chemother. 2008;62(6):1257-60. 